\title{
PINDARLEKTÜRE AN DER ACADEMIA GUSTAVIANA: Henricus Vogelmannus' ODE Prosphonetike ${ }^{1}$
}

\author{
Janika Päll
}

\section{Zwei Gratulationen zu P.Goetschenius Christognosia}

Im Korpus der 1633 in Tartu (Dorpat) gedruckten Materialien findet sich unter Nr. 23

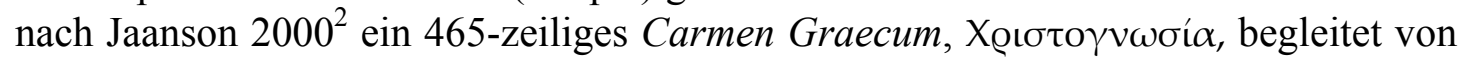
einer kleinen Einleitungsode. Der Autor dieser Oden war Petrus Goetschenius, der Professor der griechischen Sprache an der Academia Gustaviana, der später, am 2. Januar 1633 zum Lizentiat der Theologie promovierte (Tering 1978: 106/118, Piirimäe 1982: 60). Dem Titelblatt nach hat er im September 1632 seine Oden öffentlich vorgetragen, am 27. März 1633 wurden sie in Dorpat von Jacobus Pistorius gedruckt (Goetschenius 1632, auch Jaanson 2000: 119). Nach dem epischen Gesang finden sich zwei kleine Vers-Begrüssungen, zuerst eine Ode prosphonetike von Henricus Vogelmannus auf Griechisch, gefolgt von 12 lateinischen Hexametern aus der Feder des poeta laureatus Johannes Caspar Merclinus. Ob die Gratulationen zusammen mit Goetschenius' Werk im September 1632 öffentlich vorgetragen wurden, wissen wir nicht. Da die Autoren der Gratulationen den Inhalt der Goetschenius-Ode (ihrem Text nach) schon kannten und als eine ex tempore Schöpfung, mindestens im Falle der Vogelmannusode wegen der komplizierter Metrik nicht glaubhaft wäre, wurden die Gratulationen wahrscheinlich ${ }^{3}$ erst nach dem Vortrag geschrieben. Terminus ante quem bleibt der 27. März 1633. Das lateinische Gedicht des Merclinus gratuliert dem Goetschenius zur Bewältigung seiner Arbeit, deren Wichtigkeit vor allem darin liegt, dass er das Griechische verwendet (Z. 9-10: Non igitur vanam, GOETSCHENI, sumis opellam, // Occultas monstrans Graecorum dogmate Syrtes). Diese Gratulation scheint der Form und dem Inhalt nach typisch und wird hier beiseitegelassen, wie auch Goetschenius' längeres Werk, das eine gesonderte Behandlung verdient.

Die griechische Begrüssungsode von Henricus Vogelmannus (siehe: Appendix 2 mit Text und Übersetzung in die deutsche Sprache) ist in der triadischen Struktur (Strophe, Antistrophe und Epode) geschrieben, die auch im 17ten Jahrhundert meistens mit pindarischen Oden assoziiert wurde. Der hohe Pindarstil, wie auch die

\footnotetext{
${ }^{1}$ Der Artikel beruht auf meinem Vortrag vom 2. Dezember 2000, an der Konferenz Studia classica rediviva in Tartu. Er verdankt viel der langjährigen Arbeit der Forscher an der Universität und der Abteilung der alten Drucke und Handschriften an der Universitätsbibliothek. Ich danke herzlich allen, die mich beraten und mir geholfen haben, besonders Frau Ene-Lille Jaanson, Herrn Arvo Tering und Frau Kristi Viiding, wie auch Frau Jana Orion und Herrn Martin Steinrück (Universität Fribourg).

${ }^{2}$ Kopie als R.Acad.Dorp.1633: 33, das Original findet sich in Tallinn (Akadeemiline Raamatukogu).

${ }^{3}$ Diese Meinung ist jetzt von Frau Kristi Viiding bestätigt worden. Nach ihrem Ergebnissen ist das beste Beispiel dafür Lugubris ejulatio von Elias Gräntzinn (R. Acad. Dorpat. 1637: 26, Nr. 124 nach Jaanson 2000), eine Trauerpredigt zum Tod des Gustavus Adolphus, die schon im November 1634 vorgetragen, aber erst viel später, 1637 gedruckt wurde. Eine der Gratulationen zu dieser Predigt stammt vom poeta laureatus Laurentius Ludenius, der zur Zeit des öffentlichen Vortrags noch an der Universität Greifswald arbeitete und der erst Ende 1635 nach Dorpat gekommen ist (Tering 1978: $115)$.
} 
Wahl des ungewöhnlichen und komplizierten lyrischen Metrums ${ }^{4}$ sollen ihrerseits die Wichtigkeit der Gelegenheit, nämlich Goetschenius' Schöpfung einer langen theologischen Ode in griechischen Hexametern unterstreichen und können als besondere Anerkennung des neuen Universitätsprofessor betrachtet werden (vgl. Opelt 1978: 375). Wie und ob wir aufgrund dieser Ode von einer Pindartradition an der Academia Gustaviana sprechen können, wird im folgenden untersucht.

\section{Pindar als Vorbild des Vogelmannus}

Schon die triadische Struktur der Ode und ihr daktyloepitritisches Metrum verweisen auf Pindar. Um sein metrisches Vorbild noch durchsichtiger zu machen, hinweist Vogelmannus gleich am Anfang seiner kurzen Ode auf eine Zeile aus der Antistrophe

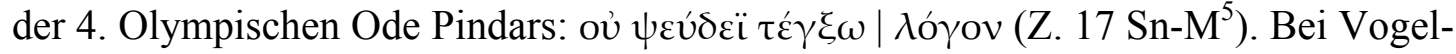

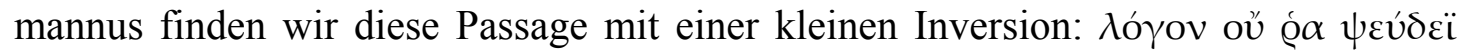
$\tau \varepsilon ́ \gamma \xi \omega$, so dass sie beinahe genau mit dem Metrum des Anfanges der ersten Zeile der

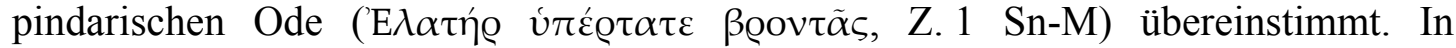
derselben Strophe gibt es auch andere Pindarnachahmungen oder Zitate aus Oden, welche Dichtung und Dichter behandeln. Aus der ersten pythischen Ode kommt wahrscheinlich die Betonung der Wahrheit des Wortes am Anfang der

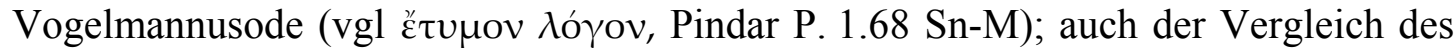
Dichters mit dem Zimmermann ( $\tau \varepsilon ́ \kappa \tau \omega v)$, bei Vogelmann Z. 9 ff., hat manche pindarische Parallelen (z.B. P. 3.113 und N. 3.4 Sn-M), das ganze Bild des Gemachs mit goldenen Säulen (Z. 9-15 bei Vogelmannus) stammt aus dem Anfang der sechsten

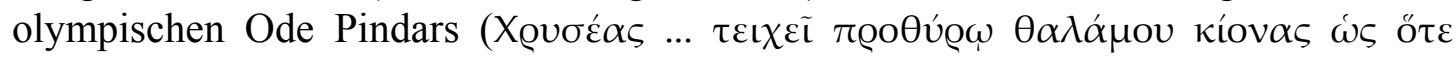

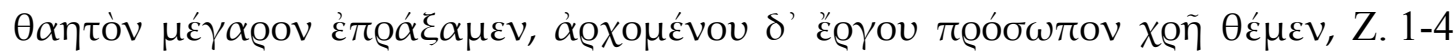
Sn-M). Danach haben wir nicht mehr viel mit Pindar zu tun (abgesehen von der Diskursform, dem Lob des Dichters, und einigen Dialektnachahmungen). In der Antistrophe und Epode folgt der Text weiter seiner eigenen Logik als Lob des christlichen Dichters und seiner Dichtung, und verweist meistens auf die Christognosia des Goetschenius, seines Adressaten ${ }^{6}$.

Als Vorbild ist Pindar in dieser Zeit nicht unbekannt. In Louvain ehren zwei pindarische Oden des Jesuiten Petrus Bovillius aus dem 1599/1600 und 1605 das Erscheinen bedeutender wissenschaftlicher Werke (Opelt 1978: 374-5), aber es wird kein bestimmtes Epinikion imitiert (ibid.: 380). In Dorpat scheint Pindar aber nicht besonders bekannt gewesen $\mathrm{zu}$ sein: zwischen 1632 und 1636 zitieren die Dissertationen, die Beispiele der alten Dichter oft benützen, Pindar beinahe nicht. Es

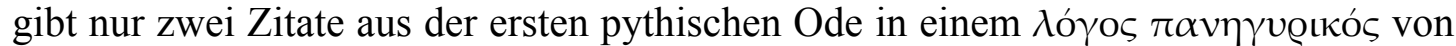
dem Adressat unserer Oden, dem Petrus Goetschenius (Jaanson 2000 Nr. 22) ${ }^{7}$. Ebensowenig gibt es im obengenanntem Korpus (nach meiner Kenntnis) andere

\footnotetext{
${ }^{4}$ Siehe unten, v.a. unter Schema 1 und 2. Was die Kompliziertheit des Metrums angeht, so hat es hier mehr mit der Konnotation des Pindarstils und seiner Metra als komplexe und als nur in Sonderfällen vorkommende Gebilde zu tun: in der Tat hat Vogelmannus für seine Ode den fast einfachsten Triadentyp Pindars gewählt.

${ }^{5}$ Nach Snell-Maehlers Pindar-Ausgabe, wie auch in weiteren Zitaten, wo nicht anders vermerkt wird.

${ }^{6}$ Diese Tradition, mit wenigen Zitaten auf das Vorbild hinzuweisen und dann weiter in eine andere Richtung zu gehen, kennen wir bereits von Horaz (z.B. seine Kleopatra-Ode carm. I.37 und Alkaios 332V.). Diese Methode erklärt z.B. Fr. Cairns in seiner Analyse der Horazischen Hermes-Oden (Alcaeus' Hymn to Hermes, P.Oxy.2734 fr.1 and Horace Odes 1.10. - QUCC n.s.13, 1983, 29-35).

${ }^{7}$ R. Acad. Dorp. 1633:24, das Original findet sich in Tallinn (Eesti Akadeemiline Raamatukogu).
} 
Oden, die pindarische metra benützten ${ }^{8}$. In dieser Periode finden sich meistens Oden in elegischen Distichen oder Hexametern. Das Profil der in den Jahren 1632-1636 ${ }^{9}$ benützten Metra zeigt die Verhältnisse zwischen lateinischen und griechischen elegischen Distichen, Hexametern und anderen Metra.

\section{Schema 1.}

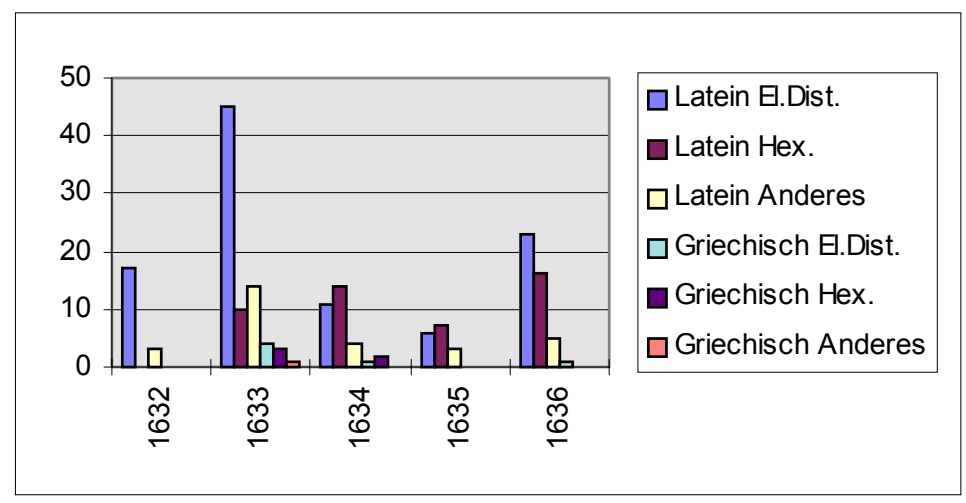

Anzahl der Oden nach Jahren

Tabelle 1: Anzahl der Oden

\begin{tabular}{|r|l|l|l|l|l|r|r|}
\hline & Latein & & & Griech. & & & \multicolumn{1}{l|}{} \\
\hline & El.Dist. & Hex. & Anderes & El.Dist. & Hex. & Anderes & \\
\hline 1632 & 17 & 0 & 3 & 0 & 0 & 0 & 20 \\
\hline 1633 & 45 & 10 & 14 & 4 & 3 & 1 & 77 \\
\hline 1634 & 11 & 14 & 4 & 1 & 2 & 0 & 32 \\
\hline 1635 & 6 & 7 & 3 & 0 & 0 & 0 & 16 \\
\hline 1636 & 23 & 16 & 5 & 1 & 0 & 0 & 45 \\
\hline$S$ & 102 & 47 & 29 & 6 & 5 & 1 & 190 \\
\hline
\end{tabular}

In allen Jahren dominiert das Latein. In Jahren 1632 und 1635 haben wir sogar überhaupt keine griechischen Gedichte. Von den 190 Gedichten sind die meisten lateinische elegische Distichen (54\%) und Hexameter $(25 \%)^{10}$. Das Griechische wird sehr wenig benutzt ${ }^{11}$ (ca. 6\%, davon 3,2\% in Distichen und 2,6\% in Hexametern). Unter andere Metra finden sich meistens lateinische Distichen, wo der erste Vers ein Hexameter, der zweite ein Enoplion oder Jambus ist, aber auch bei Horaz vorkommende Strophen, meistens die kleine sapphischen Strophen.

\footnotetext{
${ }^{8}$ Den Namen Pindars treffen wir noch einmal an, im Titel (Anapaesticum Pindaricum) der Gratulation für Georg Mancelius von Johann Skytte (R. Acad. Dorp. 1632: 11, Nr. 17 nach Jaanson 2000). Auch hier scheint es, dass diese Erwähnung eher die Funktion hat, die Anapeste durch den Namen Pindars aufzuwerten.

${ }^{9}$ Nach der Situation im Dezember 2000. Die Arbeit an der Erweiterung des Profils geht weiter.

${ }^{10}$ Nach den Angaben von Marju Lepajõe bevorzugen sogar 90\% der Autoren im ganzen Korpus der Gelegenheitsdichtung vom Dorpat elegische Distichen (Lepajõe 1992: 90), nach Anzahl der Gedichte wäre die Prozentzahl wahrscheinlich geringer. Anderseits könnten sich die Abweichungen auch mit der Verschiedenheit der Auswahl erklären: zusammen mit den Sammlungen von Gelegenheitsdichtungen, sind hier auch die Gratulationen und die am Anfang von Reden gefundene Gedichte mitgezählt.

${ }^{11}$ Nach den Angaben von Frau Kristi Viiding kommt später mehr Griechisches vor.
} 
Schema 2.

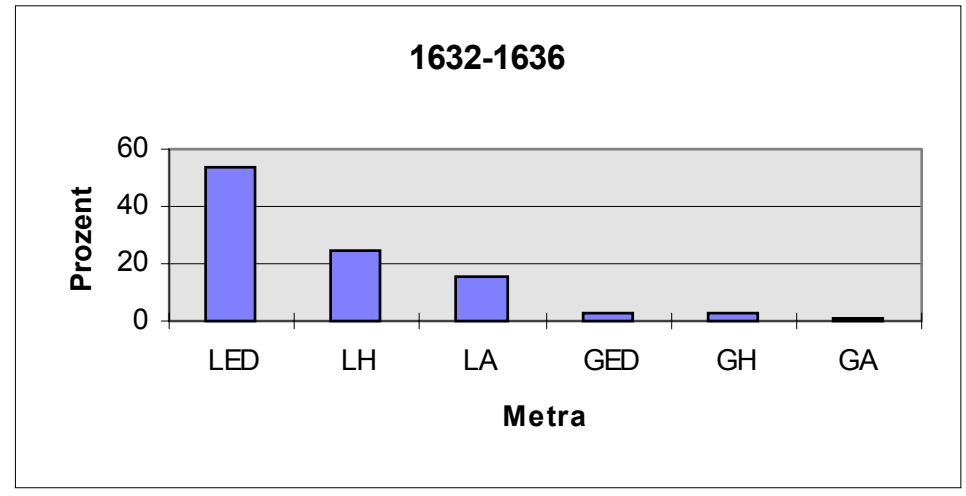

Schema 2. Die Prozentsatz der benutzten Metra zwischen 1632-1636

LED - Latein: elegische Distichen; LH - Latein: Hexameter; LA - Latein: Anderes; GED Griechisch: elegische Distichen; GH - Griechisch: Hexameter; GA - Griechisch: Anderes.

In diesem Kontext scheint es überraschend, dass Vogelmannus als Einziger überhaupt pindarische metra benützt hätte. Doch handelt es sich nicht um eine genaue Kopie oder treue Nachahmung der pindarischen Metrik. In der Vogelmannusode finden sich einige kleine Abweichungen von der übrigen Überlieferung, die zeigen, dass Pindar in 1632 in Dorpat anders gelesen wurde als heute in Tartu, Paris oder Urbino, oder auch als im Mittelalter und bei den europäischen Editoren des 16.-17. Jahrhunderts. Eine Antwort auf die Frage, in welcher Weise er sein metrisches Vorbild benutzt hat, kann uns auch zeigen, wie gut Vogelmannus' Pindar-Kenntnis war.

\section{Die strophische Struktur der Oden}

Die Pindar-Tradition von Anfängen bis in unsere Zeit kann in die folgenden Etappen eingeteilt werden (nach Irigoin 1952):

5. Jh. v.Chr. Pindar, erste Ausgabe

4. Jh.v.Chr. metagrammatismos (Umschrift in das ionische Alphabet)

2. Jh. v.Chr. Edition des Aristophanes von Byzanz, seine Kolometrie

Ende 2. Jh. n.Chr. Wahl der Epinikien (Umschrift: Papyrusrolle - Codex)

ca. 400 n.Chr. Zwei Archetypen: Ambrosianus (A): Vaticanus (andere)

ca. 500 n.Chr. Zwei Versionen des Vaticanus : verkürzte und ganze

ca. 1000 Transliterationen

1320-1330 scholia metrica von Demetrios Triklinios

1513 und später erste gedruckte Ausgaben

1811 Ausgabe von A. Boeckh, neue Kolometrie

Pindars 4. olympische Ode ist in den Handschriften und in den ersten gedruckten Ausgaben in einer Kolometrie (d.h. Einteilung in metrische Kola) dargestellt, die nicht mit der Kolometrie heutiger Ausgaben und angeblich auch nicht mit der Kolometrie Pindars übereinstimmt. Diese traditionelle Kolometrie ist von Aristophanes von Byzanz auf uns gekommen. Erst seit A. Boeckh's Ausgabe (1811) 
haben die Philologen neue Interpretationen der Kola gesucht und auch gefunden (siehe Lomiento 1999: 63-84 und Appendix 1: Einteilung der Pindarode nach scholia metrica vetera und Boeckh's längere Kola). In Vogelmannus' Ode ist jedoch die alte Kolometrie zu sehen, welche anders als die modernen Ausgaben die Strophe und Antistrophe auf 15 Kola und die Epode auf 12 Kola verteilt (Appendix 2).

Die erste Frage, ob Vogelmannus seine eigene Pindarhandschrift hatte, oder eine gedruckte Pindar-Ausgabe benutzte (es gibt etwa 20 vor Vogelmannus' Zeit, siehe: Literatur B.) kann durch die Analyse der Metrik beantwortet werden. Während die Längen der Kola bei Vogelmannus und in den ersten gedruckten Ausgaben immer dieselben sind, zeigt die genaue Analyse des metrischen Schemas, dass einige Kola in anderer Weise interpretiert werden als in heutigen und damaligen Pindartexten: die Quantitäten, die Beachtung der kurzen und langen Silben, sind machmal beim Vogelmannus verschieden. Es wäre ja möglich zu sagen, dass Vogelmannus die Metrik schlecht kannte oder unsorgfältig war. Aber das wäre zu billig. Daher müssen auch andere Erklärungen gesucht werden.

\section{Die Abweichungen in der metrischen Struktur der Oden}

Die metrischen Besonderheiten der Vogelmannusode sind (siehe auch Appendix 2):

\section{Wortende am Kolonende}

Vogelmannus weiss, dass das Wortende nicht immer mit dem Ende des Kolons zusammenfällt. Die überschneidenden Wörter finden sich jedoch nicht in denselben Positionen wie in den Pindarausgaben (in Pindar O. IV cod. ${ }^{12}$ : Str. Z. 11, 14; Ant. Z. 1; Vogelmannus aber: Str. Z. 12; Ant. Z. 10 und 12; Ep. Z. 2, 10).

\section{Beachtung des Hiats}

Vogelmannus hat eine Vorliebe für den Hiat: Bei Pindar O. IV tritt der Hiat 4mal am Kolonende auf (Str. Z. 3, 9, 12; Ant. Z.3) und einmal mitten im Kolon (Ep. Z. 8, siehe: Lomiento 1999: 65). Auch bei Vogelmannus gibt es 4 Hiate am Kolonende (Str.Z.1, 3; Ant.Z.2, 13), aber er verwendet den Hiat auch oft in der Kolonmitte, es

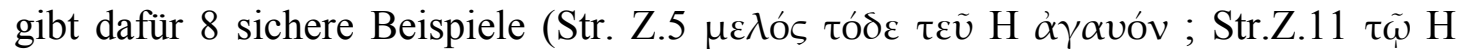

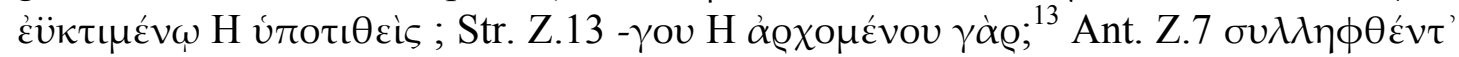

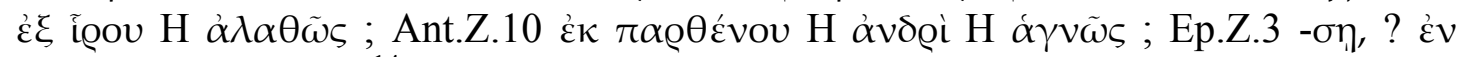

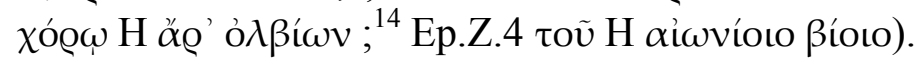

\section{Betrachtung von muta cum liquida}

Auch muta cum liquida wird von Vogelmannus anders betrachtet als von Pindar. Bei Pindar sind die mit muta cum liquida geschlossenen Silben öfter lang als kurz

\footnotetext{
${ }^{12}$ Mit cod. wird die Kolometrie der codices und der gedruckten Ausgaben bis Boeckh bezeichnet, was den Vergleich zwischen zwei Oden erleichtern soll.

${ }^{13}$ Wir wissen nicht, ob hier eine lange Silbe wie in der Antistrophe gemeint ist, und deswegen auch


dass der kurzen Silbe der Strophe eine Länge in der Antistrophe antwortet.

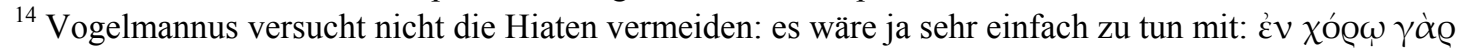
ò $\lambda \beta i ́ \omega v$.
} 
gemessen (6 zu 1, lang ist dem $\mathrm{mcl}$ vorgehende Silbe in Str. Z. 6 d $\varepsilon \dot{\theta} \lambda \omega v$, Z. 9

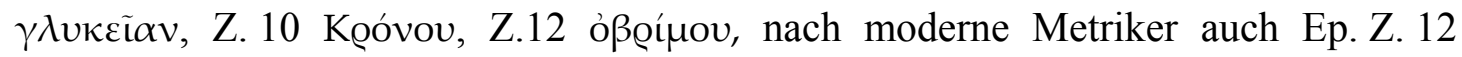

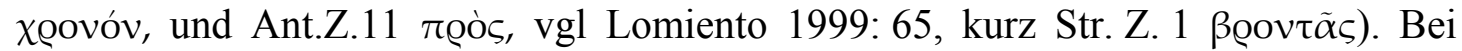
Vogelmannus werden die Silben vor der Kombination von Okklusiven und Liquiden eher kurz denn als lang gemessen. Nur einmal, in Str. Z. $14 \alpha \dot{\alpha} \gamma \lambda \alpha o ́$, ist die Silbe

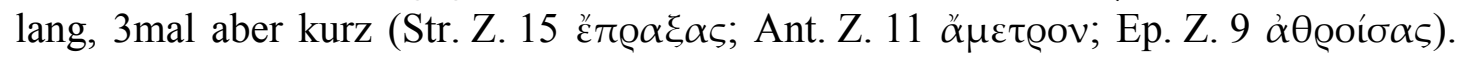

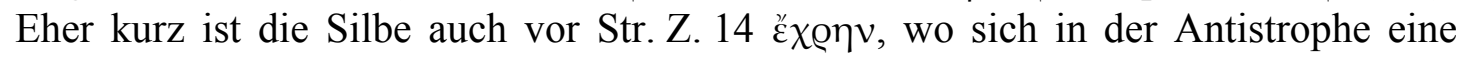
kurze Silbe findet.

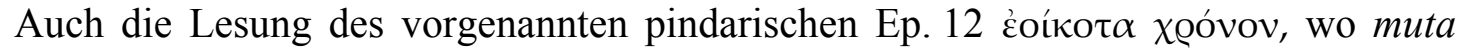
cum liquida bei modernen Metrikern als lang betrachtet wird (siehe Lomiento

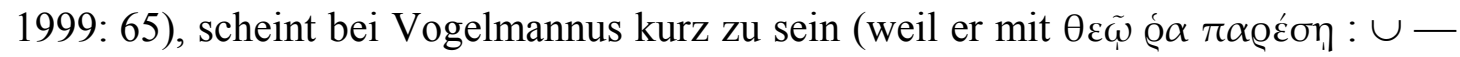
$\cup \cup \cup$ - antwortet, mit Kürze statt Länge. Es gibt noch eine Zeile, Vogelmannus'

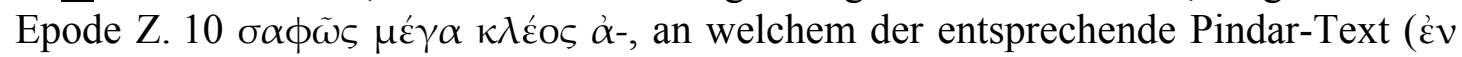
$\dot{\alpha} v \delta \varrho \alpha ́ \sigma \iota v \pi \mathrm{\prime} \iota \alpha \hat{\imath})$ in modernen Ausgaben üblicherweise als $\cup-\cup \equiv \cup \cup-$ interpretiert wird (Snell-Maehler 1988: 16). Wie mehrere Handschriften, die scholia metrica und die alten Ausgaben ${ }^{15}$, die Pindar-Text ohne $n$-ephelkystikon bieten und lesen (wie auch Lomiento 1999: 65), so könnte Vogelmannus hier die muta cum liquida als Kürze betrachten, wie das bei ihm auch sonst meistens der Fall ist.

Es ist also möglich, dass Vogelmannus die pindarische Metrik auf eine andere Weise verstand, und Silben vor muta cum liquida fast immer als Kürzen interpretierte.

\section{Verschiedene Lesarten, Abweichungen}

Manche Abweichungen von der heute als richtig betrachteten Metrik lassen sich mit der Verschiedenheit der von Vogelmannus benutzte(n) Ausgabe(n) erklären:

a. Das metrische Schema in Vogelmannus Epode Z. 11 ist: $\cup \cup-\cup \cup(\cup)-\cup \cup \cup$. Für die entspechende Zeile Pindars (O. IV. Z. 27 Sn-M) wird heute eine andere Lesart vorgezogen als damals: die nur in der Vatikanhandschrift A und den scholia metrica vetera gegebene Lesung $\theta \alpha \dot{\mu} \alpha \kappa \iota$ wird heute als richtig betrachtet. Alle andere Handschriften und die älteren Ausgaben geben aber am Anfang des Kolons $\theta \alpha \mu \alpha$

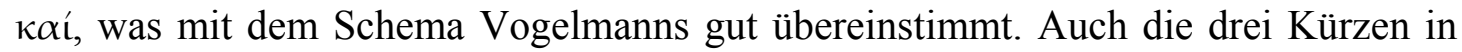

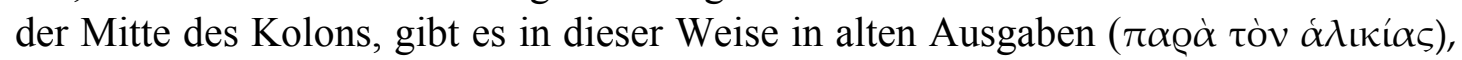

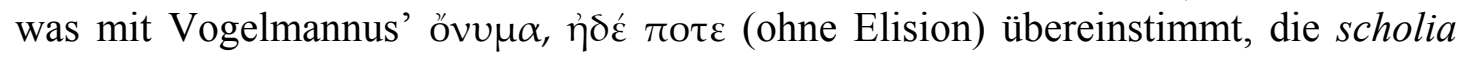

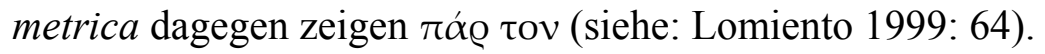

b. Das metrische Schema in Vogelmannus' Str./Ant. Z. 10 ist: $--\cup-{ }^{\mathrm{H}}-\cup^{\mathrm{H}}-$ $-\cup$ - Hier findet sich im Pindartext eine correptio metrica vor Vokal (Koóvov

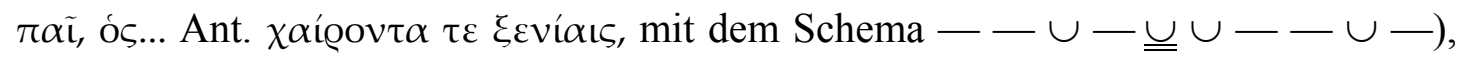

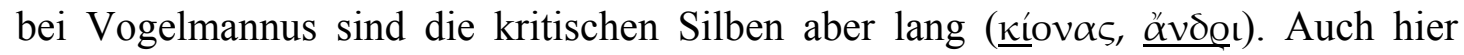
bietet sich eine Erklärung aus den alten Ausgaben an. Schon in der Ausgabe von Zacharius Callierges (1515) finden sich metrische Scholia, die dieses Kolon als

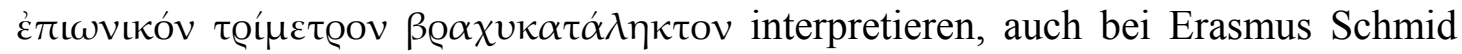
(1616) finden sich scholia metrica, wonach hier ein Trimetrum brachycatalecticum ex epitrito tertio, secundo et primo $--\cup-\ldots \cup-\cup \cup-\mathrm{zu}$ lesen sei. Auch der

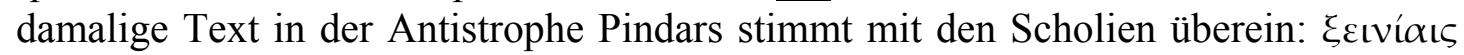

\footnotetext{
${ }^{15}$ Mit $n$-ephelkystikon: einige Handschriften (aksq $\left.\theta \tilde{\alpha}{ }^{\prime} \mu^{\prime} v^{\prime}\right)$, vielleicht Ceporinus und Benedictus; ohne: Aldus, Schmid, Calergi, Stephanus, Raphelengius, Wechel, Morel, Plantinus, Neander, Brubach, mehrere Handschriften.
} 
gibt es hier in allen vor Vogelmannus gedruckten Ausgaben. Die Nichtbeachtung der correptio metrica in Pindars Str. Z. 10 kann aber mit Vogelmannus' Vorliebe für den Hiat erklärt werden.

c. Bei Vogelmannus finden wir in Epode Z. 4 das Schema: $--\square \cup \cup \cup-$

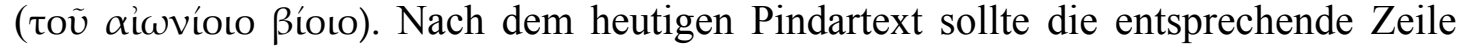

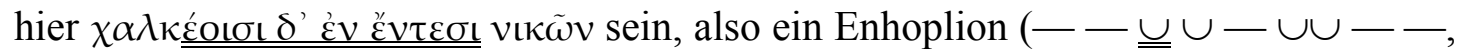
siehe: Lomiento 1999: 65). In den alten metrischen Scholien (gedruckt von Schmid) findet sich die Interpretation als ionicus a maiore, choriambus und Silbe. Diese Interpretation passt aber nicht zu dem Pindartext, an den wir heute gewöhnt sind. Es gibt aber wieder die gedruckten Ausgaben, zuerst dieselbe von Callierges, deren metrische Scholia und deren Pindartext mit Vogelmannus' Interpretation überein-

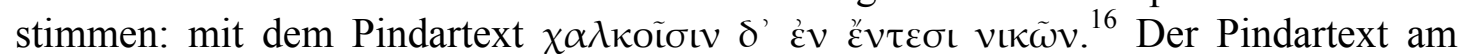

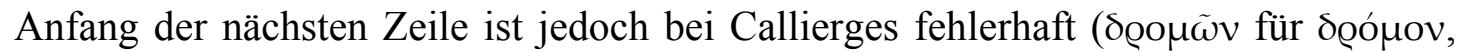
also $\cup-\cup-\cup$ pro: $\cup \cup \cup-\cup$ ), dieser Fehler findet sich auch in den Ausgaben von Brubachius und Wechel. Dass schliesst aber die Ausgaben mit den metrischen Scholien als Vorbilder des Vogelmannus nicht ganz aus, weil sich diese drei Kürzen in den metrischen Scholien bei Callierges doch noch finden.

4. Fehler. Als reine Quantitäten-Fehler kann man zwei Stellen bei Vogelmannus erklären:

a. In der Strophe: Z. 1. $\cup \cup \equiv-\cup \cup-\longrightarrow \quad$ oủ @́ $\underline{\underline{\alpha} \psi \varepsilon v ́ \delta \varepsilon i ̈ ~ . . . ~ t r i t t ~ e i n e ~ L a ̈ n g e ~}$ statt einer Kürze auf $\psi$ (in der Antistrophe stehen Kürzen, wie es auch sein sollte);

b. In der Antistrophe: Z. 5: $\cup \cup \cup \cup \equiv-\longrightarrow \tau \varepsilon \underline{\underline{\kappa \tau i ́} \sigma \tau \eta \nu . . . ~ s t e h t ~ e i n e ~ L a ̈ n g e ~}$ statt einer Kürze vor Okklusiven (in die Strophe findet sich eine Kürze, wie bei Pindar).

c. Es gibt eine Abweichung am Kolonanfang, Vogelmannus Ep. Z. 6: $\equiv-\cup \cup \cup \cup$ $\cup$, der beginnt mit $\theta \varepsilon i ́ \alpha v$ (Länge statt Kürze), weil in allen Pindarhandschriften und Ausgaben hier $\mu \varepsilon \tau \dot{\alpha}$ steht, fast immer mit einer Ligatur gedruckt.

Diese Stellen resultieren offensichtlich aus dem Problem, die passenden Wörter für das metrisches Schema zu finden, und stellen eine Notlösung dar.

Bei der Epode tritt das metrische Schema nur einmal auf, und wir haben keine Vergleichsmöglichkeit. Deshalb ist es auch nicht möglich zu entscheiden, ob die Abweichungen auf falscher Interpretation des Pindartextes beruhen oder metrische Fehler sind. Die Abweichung am Kolonende in Ep. Z. $11(=$ Z. 27 nach SnellMaehler, Kürzen statt Länge) dagegen kann nicht als Fehler oder eigene Interpretation betrachtet werden, weil es für Vogelmannus' Epoche sicher da ein Kolonende gab, wo die Länge der Silben indifferent war.

\section{Mögliches Vorbild des Vogelmannus}

Aus den oben genannten Übereinstimmungen zwischen Vogelmannus' metrischen Interpretationen und dem Pindartext bei Ceporinus, den Ausgaben der beiden Stephani; Raphelengius; Plantinus und Benedictus und den metrischen Scholien bei

\footnotetext{
${ }^{16}$ Danach findet sich dieselbe Lesart auch in der Ausgabe von Ceporinus, verschiedene Drucke von den Ausgaben der Stephani (Siehe Literatur B), Raphelengius und (nach den Angaben von Frau Jana Orion, die diese Ausgaben für mich in Göttingen überprüft hat) auch bei Plantinus, Wechel, Brubachius und Benedictus.
} 
Callierges und Brubachius, können wir schliessen, dass Vogelmannus eine dieser Pindarausgaben benutzt hat, aber nicht eine Ausgabe von Aldus, Morel oder Erasmus Schmid, wahrscheinlich auch nicht Callierges, Brubachius und Wechel. Dass er seine eigene Pindarhandschrift hatte, ist nicht glaubhaft. Nach der Pindaredition von Tycho Mommsen gibt es nur 4 Pindarhandschriften (N, E, $\left.Q^{\mathrm{s}}, \sigma \alpha^{\prime}\right)$, deren Lesungen mit der Metrik des Vogelmannus übereinstimmen. Diese sind aber zu weit von Dorpat entfernt.

Es gibt also zusammen mit den 9 verschiedenen Stephanus-Ausgaben 13 mögliche Ausgaben, die Vogelmannus hätte benutzen können (sie sind im Literaturverzeichnis $\mathrm{B}$ unterstrichen). Ob Vogelmannus seine eigene Ausgabe hatte, oder eine solche, die sich in den Bibliotheken in Dorpat oder Reval fand, wissen wir nicht.

\section{Zusammenfassung: Vogelmannus als Pindarstudent}

Wir könnten auch versuchen, Vogelmannus’ Pindarlektüre mit externen Gründen, aus seinem Leben heraus zu erklären. Es gibt aber nur wenige Angaben dazu. Vogelmannus wurde 1602, noch als ein Knabe, in Rostock, an einer Universität mit sehr guten Griechischtraditionen, immatrikuliert: es wird präzisiert, dass er keinen Eid abgelegt hat (propter aetatem non iuravit, Matrikel der Universität Rostock unter Jg. 1602, Hofmeister 1891). Später, 1611, schon volljährig, wurde er in Königsberg immatrikuliert (Erler 1910: 199). Er hat wahrscheinlich nie mit einem wissenschaftlichen Grade promoviert ${ }^{17}$, und danach wissen wir nichts bis 1631, als Vogelmannus zusammen mit Goetschenius, dem er später seine Ode gewidmet hat, in Revalschen Gymnasium arbeitete (Väänänen 1987: 299). Auch Goetschenius hat in der Universität Rostock studiert, aber später, 1616-17 (loc. supra cit.). Dann verliess Goetschenius Reval für Dorpat, Vogelmannus ist wahrscheinlich gegen Ende dieses Jahres noch in Narva gewesen (Väänänen: loc. cit.). Dann findet er sich in der Trivialschule von Dorpat als Konrektor (Liljedahl 1933: 405, nach Erpenbeck 1978: 136 zitiert), wahrscheinlich bis 1636 (Väänänen: loc. cit.). Die letzten Nachrichten haben wir von Vogelmannus aus dem Jahre 1638. In diesem Jahr verliess Georg Mancelius, noch ein Universitätsprofessor, der in Rostock studiert hat (aber 1612), Dorpat für Mitau. Auch diesem Umstand hat Vogelmannus ein Gelegenheitsgedicht gewidmet, auf Latein, nach dessen subscriptio er Rektor in der Schule des Mitau ist (Gratulationes et Vota pro felici in adventu ...DN. GEORGII $M A N C E L I I)^{18}$. Der Bericht von G. Otto über die öffentlichen Schulen Kurlands kennt aber Vogelmannus nicht. Für 1636-1639 gibt er den Namen des Rektors der Mitauischen Stadtschule nicht, nur "N.N. wird abgesetzt" (S. Otto 1904: XXXIII). Da wir die früheren und späteren Rektoren kennen, ist es möglich, das Vogelmannus hier mit N.N. bezeichnet wird. Danach gibt es von ihm keine Spur mehr.

Es handelt sich also um einen Mann, welcher getreu der damaligen Tradition mühsam nach dem Pindartext ein christliches Gedicht geschaffen hat. Auf diese Weise empfiehlt auch die spätere Konstitution der Academia Gustaviana dem Professor des Griechischen und dem Professor der Poetik, Pindar zusammen mit anderen

\footnotetext{
${ }^{17}$ Hier stütze ich mich auf die Meinung Herrn A.Terings, der mir beim Präzisieren der historischen Hintergründe und der Beziehungen zwischen den Akademikern sehr geholfen hat.

${ }^{18}$ Auch für diesen Text bin ich der Fr. K.Viiding dankbar, das Original findet sich in der akademischen Bibliothek des Lettlands in Riga.
} 
griechischen Autoren zu lesen (jeden Morgen um 7 Uhr und um 3 Uhr des Nachmittags), aber so, dass alles non impugnant christianos mores.

\section{Literatur}

\section{A. Moderne Autoren}

Cairns, Fr. 1983. Alcaeus' Hymn to Hermes, P.Oxy.2734 fr. 1 and Horace Odes 1.10. - QUCC n.s.13, 29-35.

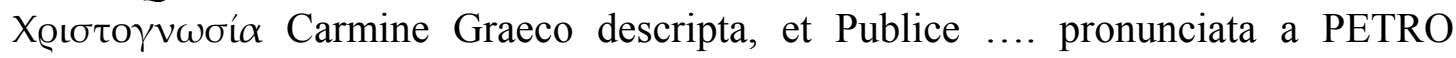
GOETSCHENIO; SS: Theologiae Licentiato.... J.Pistorii expressa, 27.d.Martii anni Christi MDCXXXIII.

Constitutiones Academiae Dorpatensis (Academia Gustaviana). Tartu Akadeemia (Academia Gustaviana) Põhikiri. 1997. Tõlkinud Kristi Sak, toimetanud Marju Lepajõe. Tartu.

Erler, G. 1910. Die Matrikel der Albertus-Universität zu Königsberg in Preussen. Bd. I. Leipzig.

Erpenbeck, Dirk 1978. Das Album des Revaler Bürgers Hans Arpenbeck, Dolmetsher der holsteinischen Gesandschaft nach Persien. - Ostdeutsche Familienkunde Bd. 8 Jhrg. 26, Heft 1, 131-8.

Gratulationes et Vota Pro felici in Curlandiam adventu VIRI Reverendi admodum, Clarissimi et Doctissimi DN: GEORGII MANCELII; SS: Theolog: Licent: .... Rigae (1634), G.Schroeder.

Hofmeister, Adolph 1891, Hrsg. Die Matrikel der Universität Rostock. Bd. 1-2. Rostock.

Irigoin, Jean 1952. Histoire du Texte de Pindare. - Études et commentaires XIII, Paris: C. Klincksieck.

Irigoin, Jean 1958, ed. Les scholies métriques de Pindare. Paris: H. Champion.

Jaanson, Ene-Lille 2000. Tartu Ülikooli Trükikoda 1632-1710. Ajalugu ja trükiste bibliograafia. (Druckerei der Universität Dorpat 1632-1720. Geschichte und Bibliographie der Druckschriften). Tartu: Tartu Ülikooli Raamatukogu.

Lepajõe, Marju 1992. Latin Poetry in seventeenth-century Estonia. - Mare Balticum Mare nostrum. Latin in the Countries of the Baltic Sea (1500-1800). Ed. by Outi Merisalo and Raija Sarasti-Wilenius. Acts of the Helsinki Colloquium, 18-21 Aug. 1992. Helsingfors: Annales Academiae Scientiarum Fennicae, ser. B - Tom. 274, 96-8.

Liljedahl, Ragnar 1933. Svensk förvaltning i Livland 1617-1634. Uppsala.

Lomiento, Liana 1999. Analisi metrica di Pindaro, Ol. 4 e 5: codici e Scholia Vetera. - La colometria antica dei testi poetici greci, a cura di Bruno Gentili und Franca Perusino. Pisa, Roma: Istituti Editoriali e Poligrafici Internazionali, 63-84.

Mommsen, Tycho 1864, ed. Pindari carmina. Berlin.

Opelt, Ilona 1968. "Zwei griechische pindarische Oden aus dem frühen 17. Jahrhundert." - Mnemosyne 21, 374-385.

Otto, Gustavus 1904. Die öffentlichen Schulen Kurlands. Mitau: J. F. Steffenhagen und Sohn.

Piirimäe, Helmut 1982, koostaja. Tartu Ülikooli Ajalugu I. 1632-1798. Tallinn: Valgus. 
Snell, Bruno; Maehler, Herwig 1984, edd. Pindarus. Pars I. Epinicia. Leipzig: B. G. Teubner.

Tering, Arvo 1978, verfasst, übersetzt und kommentiert von. Tartu Ülikooli (Academia Gustaviana) Senati Protokollid [=Konsistoriumsprotokolle der Universität Dorpat (Academia Gustaviana) 1632-1656. I (1932-1634)]. Tartu: Publicationes Bibliothecae Universitatis Litterarum Tartuensis IV.

Tessier, Andrea 1989. ed. Scholia metrica vetera in Pindari carmina. Leipzig: B. G. Teubner.

Väänänen, Kyösti 1987. Herdaminne för Ingermanland I. Helsingfors.

\section{B. Pindar-Ausgaben vor 1633}

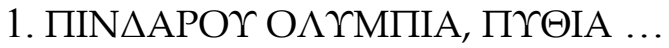

Aldus, Venetiis 1513.

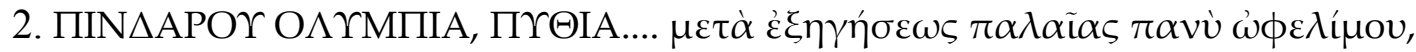

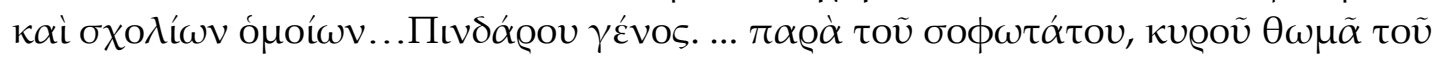

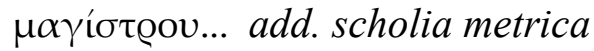

Callierges, Romae 1515.

3. ПIN $\triangle \mathrm{APO} \Upsilon \mathrm{O} \Lambda \Upsilon \mathrm{M} \Pi \mathrm{A}, \Pi \Upsilon \Theta I A . .$.

Ceporinus, Basileae 1526, Neudr.1556?

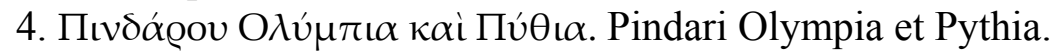

Parisiis ex Officina Christiani Wechel 1535.

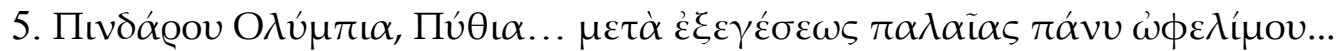

Pindari Olympia, Pythia...

Francoforti opera et impensa Petri Brubachii 1542.

6. Pindari Olympia...

Christophorus Plantini. Antverpiae 1549.

(6a. nicht vollständig) Pindari Aristologia...

M. Neander, Basileae 1556.

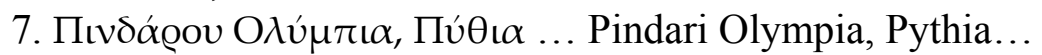

Parisiis M.D.LVIII Apud Guilielmum Morelum.

8. Pindari Olympia, Pythia, Nemea, Isthmia. Caeterorum octo Lyricorum carmina, Alcaei, Sapphus... Nonnulla etiam aliorum.

H.Stephanus, Genavae $1560^{1}$.

Editio II Graecolatina ...

H.Stephanus, Genavae $1566^{2}$.

H.Stephanus, Genavae $1586^{3}$ (cum notis Casauboni).

Editio IIII Graecolatina ....Lugduni, apud J.Pillehotte M.D.XCVIII.

H.Stephanus, Lugduni Batavorum $1598^{4}$.

H.Stephanus $1612^{5} ; 1624^{6}$.

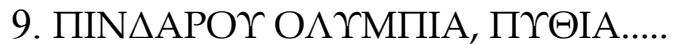

Lugduni Batavorum ex officine Plantiane apud Franciscum Ralphelengium M.D.XC.

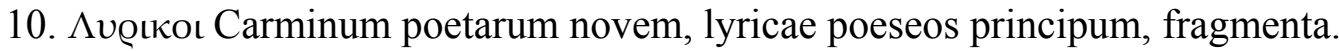

Alcaei, Sapphus... Pindari...

Apud Hieronymum Commelinum, Elect. Palat. Typographum. Anno

MDXCIIX. Illustribus viris ... H.Stephanus, Heidelberg 1598. 


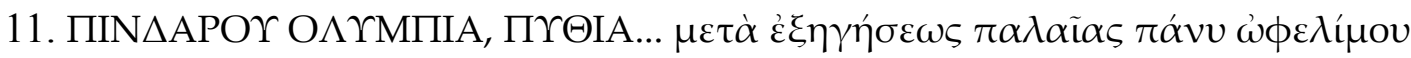

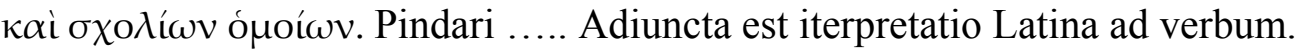

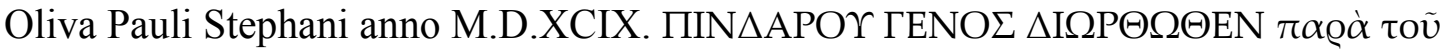

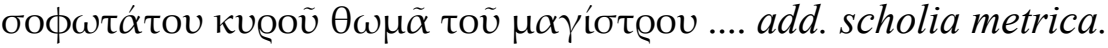

P.Stephanus, Genavae 1599.

12. ПIN $\triangle \mathrm{APO} \Upsilon \Pi \mathrm{IEIO} \triangle \mathrm{O} \Sigma$.... add. metrorum ratio.

E.Schmid, Vitebergae 1616.

13. ПIN $\triangle$ APO $\Upsilon$ ПEPIO $\triangle \mathrm{O} \Sigma$ Pindari Olympia, Pythia....

J.Benedictus, Salmurii 1620 . 


\section{Appendix 1}

Pindar O.IV.

Text und metrisches Schema nach der antiken Kolometrie gestattet (L.Lomiento ${ }^{19}$ )

Str.

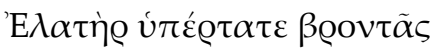

$\dot{\alpha} \kappa \alpha \mu \alpha \nu \tau o ́ \pi$ oठо

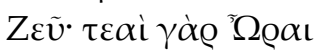

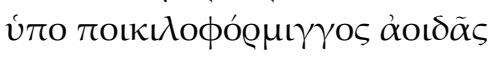

$\varepsilon \dot{\lambda} \iota \sigma \sigma o ́ \mu \varepsilon v \alpha i ́ \mu^{\prime} \varepsilon^{\prime \prime} \pi \varepsilon \mu \psi \alpha \nu$

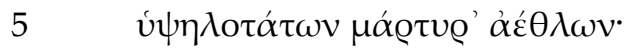

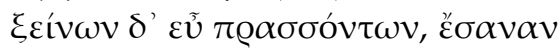

$\alpha \hat{\jmath} \tau \iota \kappa^{\prime} \dot{\alpha} \gamma \gamma \varepsilon \lambda \hat{\prime} \alpha \nu$

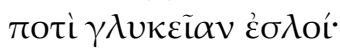

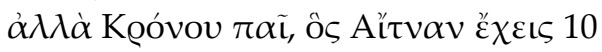

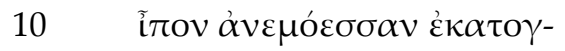

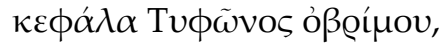

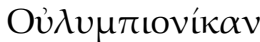

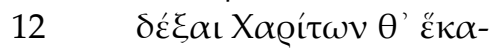

$\tau \iota \tau o ́ v \delta \varepsilon \kappa \tilde{\omega} \mu \mathrm{ov}$

Ant.

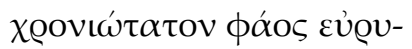

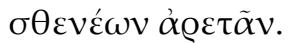

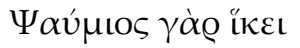

15

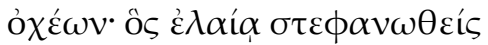

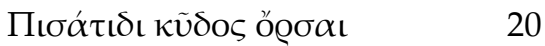

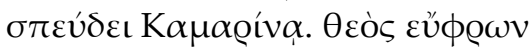

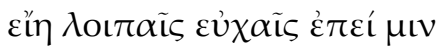

$\alpha i v \varepsilon ́ \omega, \mu \alpha \dot{\alpha} \lambda \alpha \mu \varepsilon^{\prime} v$

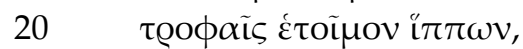

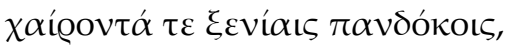

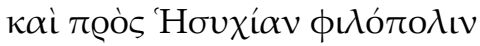

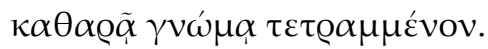

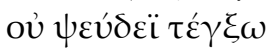

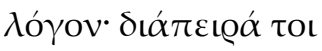

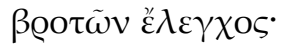

Ep.

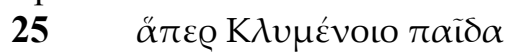

$\Lambda \alpha \mu \nu\llcorner\alpha \dot{\alpha} \alpha v \gamma v v \alpha \iota \kappa \tilde{\omega} v$

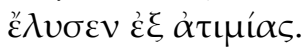

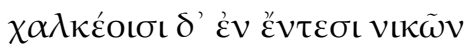

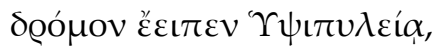

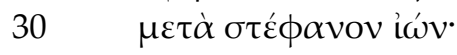

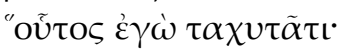

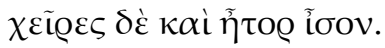

${ }^{19}$ Die Numerierung ist vereinfacht. 


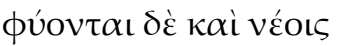

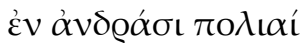

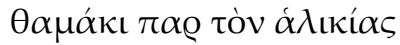

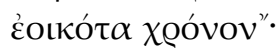

Metrisches Schema:

Str./Ant.

$$
\begin{aligned}
& \cup \cup \cup-\cup \cup-- \\
& \cup \cup-\cup \cup-\mid \\
& -\cup-\cup--\|^{\mathrm{H}} \\
& \cup \cup-\cup \cup--\cup \cup--\mid \\
& \cup-\cup \cup-\cup-a \mid \\
& --\cup \cup--\cup \cup--\mid \\
& ------\cup-u \| \\
& -\cup-\cup \cup-\mid \\
& \cup-\cup-\cup--\|^{\mathrm{H}}
\end{aligned}
$$

5

10

$$
\begin{aligned}
& --\cup-\cup \cup--\cup-\mid \\
& -\cup-\cup \cup-\cup \cup \cup- \\
& \cup---\cup-\cup-\|^{\mathrm{H}} \\
& a-\cup \cup--\mid \\
& u-\cup \cup-\cup- \\
& \cup-\cup--\|
\end{aligned}
$$

Ep.

$$
\begin{aligned}
& -\backsim \cup \cup-\cup-\cup \mid \\
& -\cup \cup-\cup--\mid \\
& \cup-\cup-\cup-\cup-1
\end{aligned}
$$

5

$$
--\cup \cup-\cup \cup--1
$$

$$
\cup \cup-\cup-\cup \cup--1
$$$$
\cup-\cup \cup \cup \cup-1
$$$$
--\left.\cup \dot{\mathrm{E}}\right|^{\mathrm{H}}-\cup-\ldots
$$$$
\cup--\cup-\cup-1
$$

$10 \cup-\cup \cup \cup \cup-1$

$\cup \cup \cup \cup-\cup \cup-1$

$\cup-\cup-\cup-\|$ 


\section{Appendix 2}

\section{Henricus Vogelmannus}

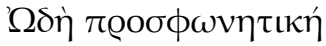

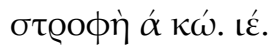

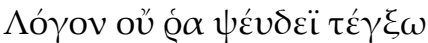

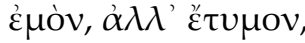

$\omega \varsigma \mu \varepsilon v \tilde{v} v \kappa \varepsilon \lambda \varepsilon v ́ \varepsilon เ$

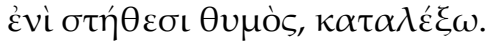

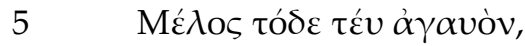

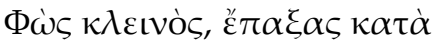

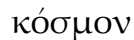

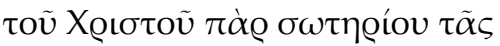

$\gamma \nu \omega ́ \sigma \varepsilon о \varsigma, \theta \alpha \lambda \alpha ́ \mu \nu$

$\kappa \alpha \theta \dot{\omega} \varsigma \theta \alpha \eta$ tòv oĩ̌ov.

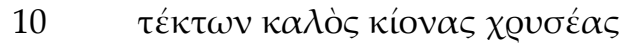

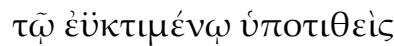

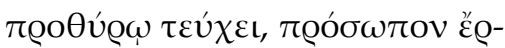

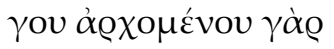

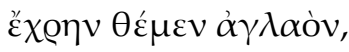

15

ö $\pi \omega \varsigma$ हैं $\mathrm{\rho} \alpha \xi \alpha \varsigma^{\circ}$

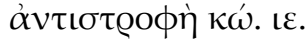

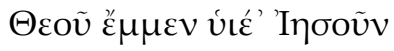

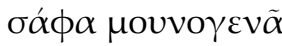

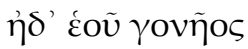

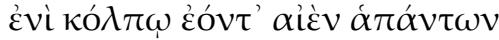

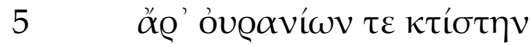

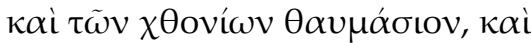

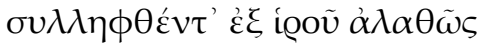

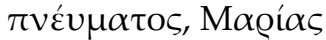

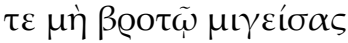

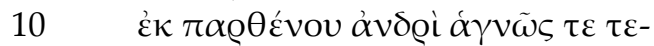

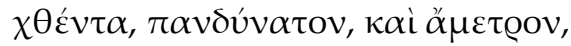

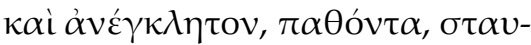

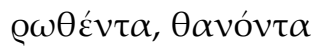

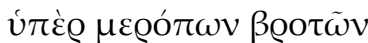

15 $\kappa \alpha \kappa \tilde{\omega} \nu \dot{\alpha} \pi \alpha \dot{v} v \tau \omega \nu$

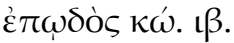

$\dot{\varepsilon} \kappa \tau \tilde{\alpha} \varsigma \kappa \rho \alpha \delta i ́ \alpha \varsigma \dot{\alpha} \pi \alpha ́ \sigma \alpha \varsigma$

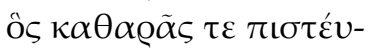

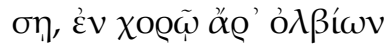

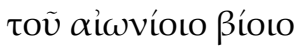

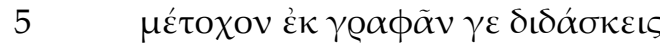




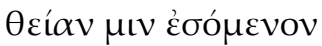

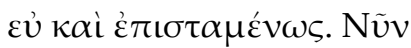

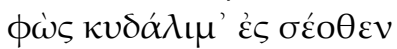

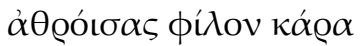

10

$\sigma \alpha \phi \tilde{\omega} \varsigma \mu \dot{\varepsilon} \gamma \alpha \kappa \lambda \hat{\varepsilon} \mathrm{\sigma} \alpha \dot{\alpha}-$

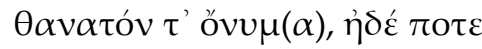

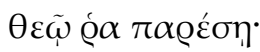

Metrisches Schema

$$
\begin{aligned}
& \cup \cup-\hat{I}-\grave{E} \dot{E}--\|^{\text {Ha }} \\
& \cup-\cup \cup u \|^{\mathrm{Ha}} \\
& -\cup-\cup-\mathrm{a} \|^{\mathrm{Ha}} \\
& \cup-\cup \cup-\cup \cup--1 \\
& \cup-\cup \cup-{ }^{\mathrm{Hs}} \underline{\cup}-- \\
& --\cup U--\cup U--1 \\
& ---\ldots-{ }^{\mathrm{Ha}} \cup--1 \\
& -\cup-\cup \cup-1 \\
& -{ }^{\mathrm{H}} \cup-\cup \cup-\cup \cup \cup-1 \\
& \cup---\cup-\cup- \\
& { }^{\mathrm{Hs}} \mathrm{H}-\cup \cup-\cup
\end{aligned}
$$

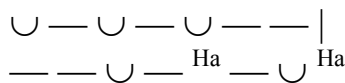$$
5
$$

10

Epode

$$
\begin{aligned}
& --\cup \cup-\cup--1 \\
& -\cup \cup-\cup-- \\
& A^{\mathrm{H} ?}-\cup-{ }^{\mathrm{H}} \cup-\cup-1 \\
& { }^{\mathrm{H}}-\square-\cup-\cup \cup-\cup \mid
\end{aligned}
$$

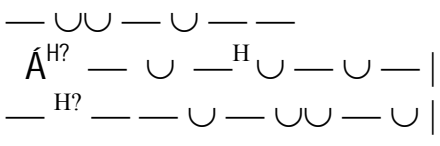

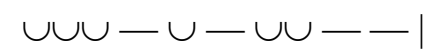
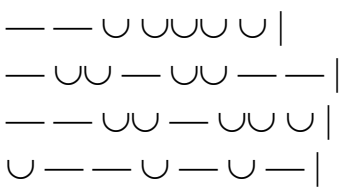

10

$$
\begin{aligned}
& \cup-\cup \text { E } \cup- \\
& \cup \cup-\cup(\cup)-\cup \cup \mid
\end{aligned}
$$

Übersetzung

Str.

Meine Rede werde ich nicht in Lüge einweichen, sondern, wie mir das Herz in der Brust befiehlt - werde ich die Wahrheit erzählen.

Deine leuchtende Melodie, ruhmvoller Mann, hast Du mit Schönheit zusammengebracht, nach dem Erkennen Christi, des Erlösers,

wie ein guter Zimmermann das wunderbare Haus mit wohlbestellten Vorhallen einrichtet, den Frauengemächern die goldene Säulen. -

Denn ist es notwendig, den begonnenen Werken ein strahlendes Angesicht zu geben, was Du auch getan hast. 
Ant.

Dass Jesus des Gottes Sohn ist, deutlich eingeboren,

und das er immer im Schoss seines Erzeugers bleibt als ein wunderbarer Schöpfer alles

Himmlischen und Irdischen;

und dass er wirklich vom Heiligen Geist empfangen ist,

geboren unbefleckt von der Jungfrau Maria,

die mit keinem sterblichen Mann sich vereinigt hat;

der allmächtig ist und unermesslich, und untadelig, der litt, gekreuzigt wurde, starb wegen der sterblichen Menschen, die alle böse sind;

Ep.

wer von ganzem reinen Herz glaubt, wird am ewigen Leben

mit dem Reigen der Heiligen teilnehmen - , lehrst Du aus den Schriften,

der wird göttlich werden, wohl weisst Du es auch.

Jetzt aber, Du ruhmvoller Mann, hast Du deutlich auf dein liebes Haupt

grossen Ruhm und einen unsterblichen Namen gehäuft,

und wirst einmal neben Gott sein. 